\title{
3D Cranio-Facial Surface Modeling from 2D CT Slices Using Fast Corner Detector
}

\author{
Menaka.R and Chellamuthu. C
}

\begin{abstract}
This paper presents a novel approach for 3D Cranio-Facial reconstruction using Fast Corner detector. The Method involves Bilateral Filtering as the preprocessing step to suppress unwanted distortions. The contours representing the bony areas of the skull are extracted using Region of interest processing.The feature points required for the point cloud generation are detected by FAST corner detector. The generated point cloud is subjected to Delaunay triangulation for surface reconstruction. The results are compared with three different 3D reconstruction methods.
\end{abstract}

Index Terms-Fast Corner Detector, point cloud, 3D Reconstruction, Triangulation

\section{INTRODUCTION}

Retrieving three-dimensional geometric information of objects fromtwo-dimensional images is one of the challenging tasks in Medical image modeling. Reconstruction of 3D surface models using 2D Computed Tomographic slices provides many advantages to the doctors in diagnosing the pathological formations and preparing the treatment plans. 3D Surface rendering is used for visualizing the shapes of 3D objects and the spatial relationships among these objects. The surfaces of objects are first defined by set of points in 3D and modeled by polygons or other geometric primitives through segmentation or surface detection. These geometrical primitives are then rendered using the conventional computer graphics techniques for geometricalobjects. The set of points extracted fromeach CT slice is referred to as point cloud where the terms "feature point" and "corner" refer to a small point of interest with variation in two dimensions. Such points often arise as the result of geometric discontinuities, such as the corners of real world objects, but these may also arise from small patches of texture.

There are numerous techniques available for point cloud generation and surface reconstruction. Some of the corner point detectors are Harris detector, Susan detector etc.

Harris. C, Stephens .M, [2] suggests that the Harris corner detector computes a matrix related to the autocorrelation function of the image and the eigen values of the resulting matrix are the principal curvatures of the Auto-correlation function. An interest point is detected if the found two curvatures are high. The main advantage of Harris operator is that the detected points are well proportioned and valid. The disadvantage is the detection accuracy can only reach one pixel.

Jie Chen, Li-hui Zou [3], describes that Susan corner detector, places a circular mask over the pixel to be tested (the nucleus). If the brightness of each pixel within a mask is compared with the brightness of that mask's nucleus, then an area of the mask can be defined which has the same (or similar) brightness as the nucleus. The disadvantage is that a fixed global threshold is not suitable and the anti-noise ability is weak.

Some of the surface reconstruction includes trilinear interpolation, Marching cubes, Delaunay triangulation etc. William. ELorensen, Harvey, [8] describes Marching Cubes technique that creates a triangular mesh to approximate the is o-surface. Algorithmworks by locating the surface in a cube of eight pixels and calculates normals. I.Fujis hiro, Y. Maeda, $H$. Sato, et al [6], extracted the tetrahedral meshes between two is osurfaces from volume datasets. Drawback is that holes will get generated in the model

Fas to Bernardini, Joshua Mittleman, [1] computes a triangle mesh interpolating a given point cloud. But, it fails to work well for large quantity of points.

The reconstruction of a surface consistent with the parallel contours is a very intriguing and challenging problem, as the construction can be non-unique due to severe topology variations or sparseness of contours. In this work, FAST corner detector is used for Point cloud generation due to its Computational time efficiency. Delaunay triangulation is employed for surface reconstruction.

\section{PROPOSED SYSTEM}

A method is proposed using FAST (Features from Accelerated Segment Test) Corner Detector to locate the interest points for point cloud generation. Delaunay Triangulation is applied to the point cloud for 3D surface Reconstruction.

\section{A. Data Acquisition}

CT slice data is generated using an X-ray source that rotates around the object. X-ray sensors are positioned on the opposite side of the circle from the X-ray source. Many data scans are progressively taken as the object is gradually passed through the gantry. They are combined together by the mathematical procedures known as tomographic reconstruction. The data are arranged in a matrix in memory. The format used by a CT machine to produce the scanned slices is in DICOM [Digital Imaging and Communication in Medicine] as shown in Fig 1. The images in 
this format contains information about the slice angle, depth etc. Normally the thickness of the slice will be $.75 \mathrm{~mm}$.

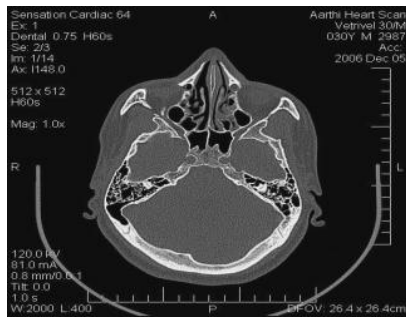

Fig 1. DICOM Image

with a pixel spacing of [ 2 X 1 double] and with a height and width of 512 X 512. DICOM format can differentiate up to 1024 gray levels whereas TIFF format can differentiate up to 256 levels proposed system is intended to have low memory requirements. Hence slices are converted from DICOM format to TIFF format, without losing the structural information.

\section{B. System Description}

The functional modules of the system have two phases namely pre-processing and reconstruction. Pre-processing involves bilateral filtering and further processing includes Slice Thresholding, ROI processing, FAST corner detection and Point cloud generation.

Surface reconstruction is performed through Delaunay triangulation. Fig 2 depicts the System architecture of the system

\section{Pre-processing}

Pre-processing is carried out to suppress unwanted distortions or enhances some image features important for further processing. Bilateral Filtering is applied to remove unwanted noise and distortions.

\section{Bilateral Filtering:}

A bilateral filter is an edge-preserving smoothing filter. It replaces a pixel's value by a weighted average of its neighbors in both space and range (pixel value). This preserves sharp edges by systematically excluding pixels across discontinuities from consideration. For the central pixel of the neighborhood ao, its new value, denoted by,
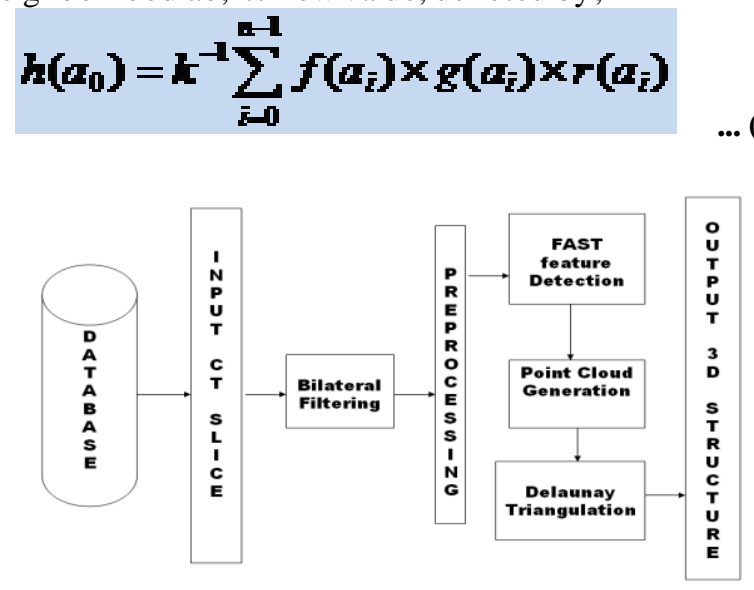

Fig 2. System Architecture

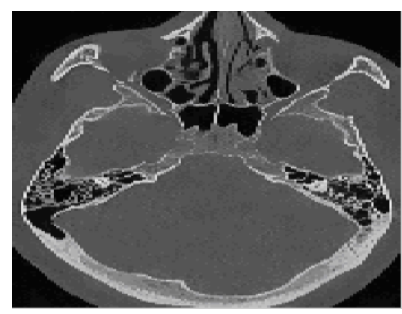

Fig 3. Bilateral Filtered Image where ' $\mathbf{g}$ ' is the domain filter and ' $\mathbf{r}$ ' is the range filter, ' $\mathbf{k}$ ' being the normalization constant. Bilateral filtered image is shown in Fig 3.

\section{E. Region of Interest (ROI) Processing}

ROI returns a region of interest within an indexed or intensity image and returns a binary image. Threshold required for ROI processing is determined by global thresholding of CT slice.

\section{Global Threshold:}

Global threshold is computed by determining the average value of the maximum and the minimum pixel value of the CT slices. Threshold range used is tabulated in Table 1 .

TABLE 1. THRESHOLD RANGE

\begin{tabular}{|c|c|}
\hline SLICE NO & THRESHO LD RANGE \\
\hline $1-215$ & $0.4-0.5$ \\
\hline $216-236$ & $0.3-0.4$ \\
\hline
\end{tabular}

ROI processing is carried out for each CT slice and the result of this process is shown in Fig 4.

\section{F. FAST Corner Detection}

The feature detector considers pixels in a Bresenham circle of radius $r$ around the candidate point. If $n$ contiguous pixels are all brighter than the nucleus by at least threshold ' $t$ ' or all darker than the nucleus by ' $t$ ', then the pixel under the nucleus is considered to be a feature.

FAST corner detector operates in two stages. It begins by using the segment test criterion for ' $n$ ' number of pixels and a convenient threshold.

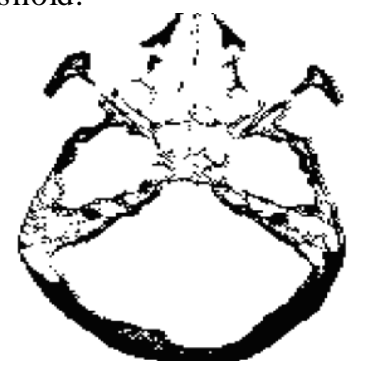

Fig 4. ROI processed image

Let $\mathbf{P}$ be the set of all pixels in the test image. $\mathbf{P}$ is partitioned into three subsets Pd (darker), Ps (similar), Pb(Brighter). Every center pixel is compared with the set of contiguous pixels around it. If all the contiguous pixels are all either darker or brighter when compared to the center pixel, then the pixel under consideration can be defined as a corner point.

FAST Corner Detector is computationally efficient and provides stable features. It has high detection rate and the 
factor of repeatability proves to be good for affine transformations.FAST corner detector is applied to the ROI detected image to locate the interest points and the result is shown in Fig 5.

\section{G. Point Cloud Generation:}

Point cloud represents visible surfaces of an object. point cloud is generated by processing all the $2 \mathrm{D}$ images required to reconstruct the three dimensional structure. To obtain the point cloud, metadata contained in the header of each individual slice is collected .The contoured slices are processed to obtain the three dimensional coordinate vertices of the points. The coordinate positions of the pixels are taken with respect to a reference point for each slice. These three dimensionalvertices represents the point cloud. The following equations are used for finding the vertices

$$
\begin{array}{lll}
\mathrm{X} \text { new } & =\mathrm{x}(\mathrm{i}, \mathrm{j}) * \text { pixelspace }+ \text { offsetx } & \rightarrow(2) \\
\text { Ynew } & =\mathrm{y}(\mathrm{i}, \mathrm{j}) * \text { pixelspace }+ \text { offset } \mathrm{y} & \rightarrow(3) \\
\mathrm{Z}_{\mathrm{k}} \quad=\mathrm{Z}_{\mathrm{k}-1} \text { - Gap }(\mathrm{K}=2 \ldots \text { No of slices }) & \rightarrow(4) \\
\mathrm{Z}_{1} \quad=\text { Offset } \mathrm{z} & \rightarrow(5)
\end{array}
$$

Offset $\mathrm{x}$ - reference point (origin) in the $\mathrm{X}$ direction

Offset y - reference point (origin) in the Y direction

Offset $\mathrm{z}$ - reference point in the (origin) in the $\mathrm{Z}$ direction.

Gap - the distance between the adjacent slices. $\mathrm{i}, \mathrm{j}$ - coordinates in $\mathrm{X}, \mathrm{Y}$ direction.

The point cloud for the CT slices is shown in Fig 6 .

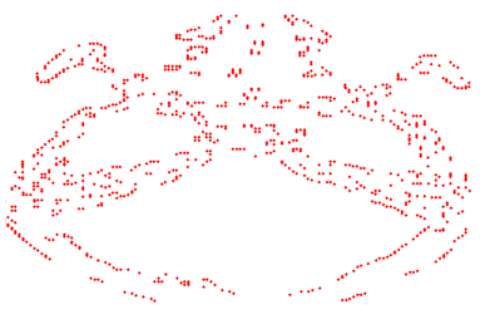

Fig 5. FAST Corner Detection

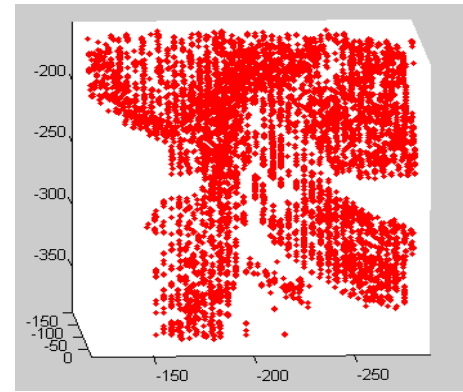

Fig 6. Generated Point cloud

\section{H. Reconstruction:}

Reconstruction is performed to obtain the $3 \mathrm{D}$ structure. Delaunay Triangulation is used in the reconstruction process . Delaunay Triangulation
A Delaunay triangulation for a set $\mathrm{P}$ of points in the plane is a triangulation DT $(\mathrm{P})$ such that no point in $\mathrm{P}$ is inside the circumcircle of any triangle in DT (P) as shown in Fig 7.

It maximizes the minimum angle of all the angles of the triangles in the triangulation. They tend to avoid skinny triangles.

Delaunay triangulation is applied to the co-ordinates obtained from the point cloud. The resultant output of this process is shown in Fig 8.

\section{RESUlTS AND DISCUSSION}

A set of 236 CT slices in axial plane has been collected from a male patient. It is subjected to both preprocessing and reconstruction phases. The final $3 \mathrm{D}$ reconstructed structure is shown in Fig 9.

The final reconstructed structure obtained by various methods is compared in Fig. 10, 11 and 12. The time taken for reconstruction using FMWP (FAST method without preprocessing) and FMP (FAST method with preprocessing) is found to be comparable with the Marching Cubes method (MCR), Sobel detector based reconstruction (SBR) and Harris detector based reconstruction.(HBR

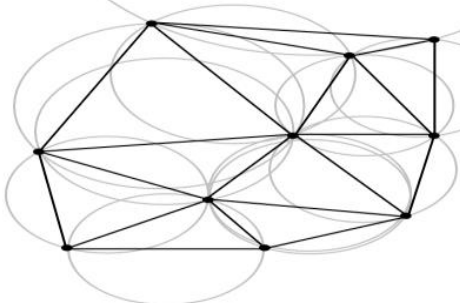

Fig 7. Circumcircle property

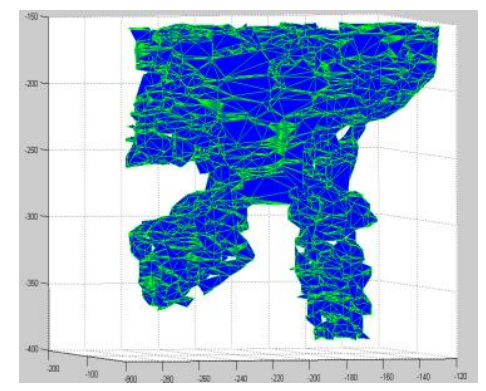

Fig 8. Delaunay Triangulation

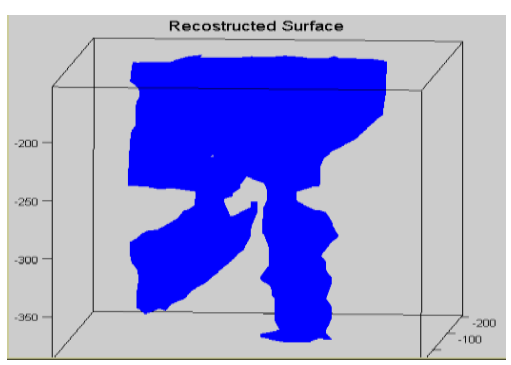

Fig 9. 3D Reconstructed Skull

Chart 1. Comparison of Reconstruction time 


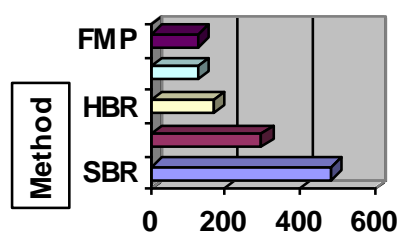

Reconstruction time (Sec)

T ABLE 2. COMPARISON OF TRIANGULAR INDICES

\begin{tabular}{|c|c|}
\hline Method & $\begin{array}{c}\text { No. of Triangular } \\
\text { Indices }\end{array}$ \\
\hline $\begin{array}{c}\text { Sobel detector based 3D } \\
\text { reconstruction }\end{array}$ & 30,784 \\
\hline $\begin{array}{c}\text { Harris Detector based 3D } \\
\text { Reconstruction ( without } \\
\text { Preprocessing ) }\end{array}$ & 8,522 \\
\hline $\begin{array}{c}\text { FAST Detector based 3D } \\
\text { Reconstruction ( without } \\
\text { Preprocessing ) }\end{array}$ & 10,348 \\
\hline $\begin{array}{c}\text { Fast Detector based 3D } \\
\text { Reconstruction (with Bilateral } \\
\text { Filtering ) }\end{array}$ & 9,617 \\
\hline
\end{tabular}

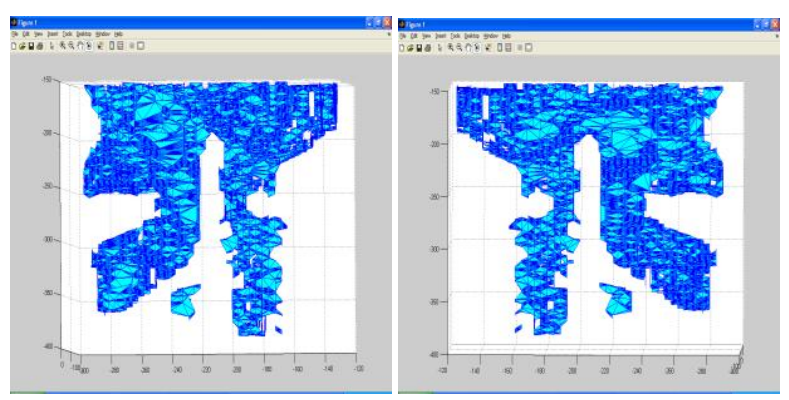

Fig 10. Final reconstructed structure by Sobel operator based Method.
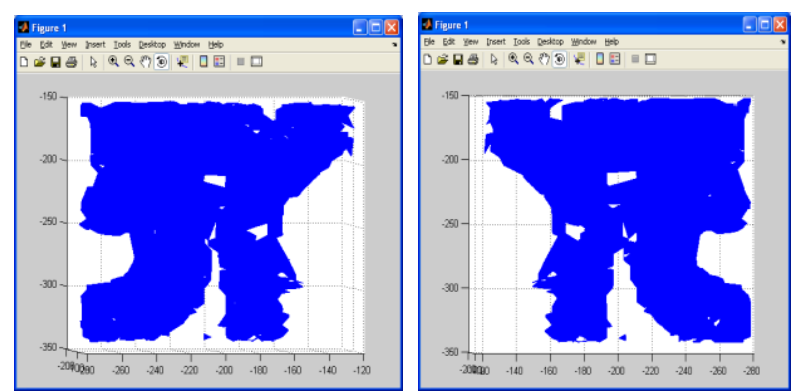

Fig 11. Final reconstructed structure by Harris detector Method.
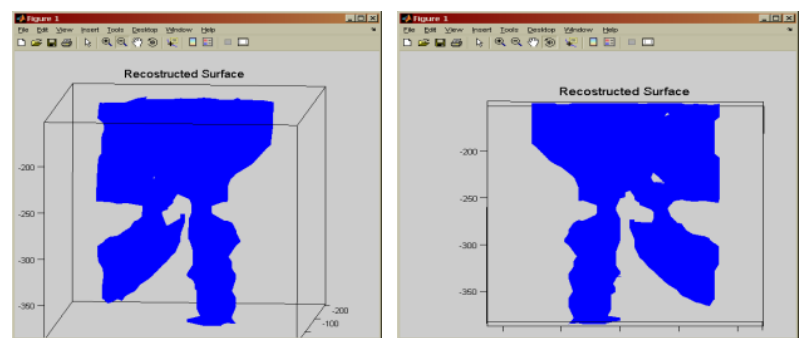

Fig 12. Final reconstructed structure by FAST detector method.

\section{CONCLUSION AND FUTURE WORK}

FAST Corner detector was applied on each CT slice for feature point detection and point cloud generation and the 3D reconstructed structure of the skull is visualized. It has been observed that the reconstruction time is reduced by $40 \%$ when compared to sobel detector based reconstruction. Also the number of triangular indices formed is reduced by $30 \%$. Reconstruction time is much reduced due to high detection rate ofFAST corner detector. FAST corner detector is applied to medical images for the first time. The work of this paper provides a new direction in the improvement of point cloud generation.

\section{ACKNOWLEDGEMENT}

The authors wish to thank RAGAS DENTAL COLLEGE, CHENNAI for providing the CT Slices and Dr. Gnanasundaram Professor Dept of Oral Medicine, SAVEETHA DENTAL COLLEGE for his several key suggestions in improving this paper.

\section{REFERENCES}

[1] Fausto Bernardini, Joshua Mittleman Holly Rushmeier $\mathrm{Cl}^{\prime}$ audio Silva Gabriel Taubin. "The Ball Pivoting Algorithm for surface reconstruction", IEEE Transactions on Visualization and Computer Graphics, 5(4), Oct-Dec, 1999, pp. 349-359.

[2] Harris, C., Stephens, M, “A combined corner and edge detector". In: Fourth Alvey Vision Conference, Manchester, UK, pp.147-151, 1988.

[3] Jie Chen, Li-hui Zou, Juan Zhang and Li-hua Dou., "The Comparison and Application of Corner Detection Algorithms",Journal of Multimedia, Vol. 4, no. 6, December 2009.

[4] E. Rosten and T. Drummond ,"Machine learning for high-speed corner detection", ECCV 2006.

[5] Sander Koelstra, Ioannis Patras, "The fast-3D spatio-temporal interest region detector", 10th Workshop on Image Analysis for Multimedia Interactive Services 2009.

[6] .Fujishiro, Y. Maeda, H. Sato, et al., "Volumetric data exploration using interval volume",IEEE Tran. Visualization and Computer Graphics, 1996, Vol. 2, pp. 144-155.

[7] Wenlian Cheng, Zhiya Qian, Lina Lu,'Research On Medical Image Three Dimensional Visualization System".

[8] William E. Lorensen, Harvey E.Cline "Marching Cubes: A High Resolution 3D Surface Construction Algorithm”, - SIG '87 IEEE/ICME International Conference on Complex Medical Engineering, 2007.

[9] Wu Z, Sullivan JM., "Multiple material marching cubes algorithm", International Journal for Numerical Methods in Engineering 2003; 58(2):189 -2007.

Mrs $\mathbf{R}$ Menaka, received the master degree in Engineering in Applied Electronics from Anna University, Chennai., India. Bachelor of Engineering in Electronics and Communication Engineering from Madurai Kamaraj University, India. Currently she is currently working as Associate Professor in School of electronics, VIT University, Chennai. and also a research Scholar in Anna University, Chennai, India. Her areas interest are Image processing, Neural Networks and fuzzy logic.

Dr C Chellamuthu received his $\mathrm{PhD}$ degree in Power Electronics from IIT, Chennai, India. After working as Associate lecturer in GCT, Coimbatore, India, he joined Anna University, Chennai, India.as Lecturer. He served there as Asst professor ,professor and as Head of Department of Electrical and Electronics Engineering. He is currently working as professor at RMK College of Engineering, Chennai, India. He has published his works in Several International and National journals, pioneered several new research directions, made a number of landmark contribution in his field. His areas of interest are power Electronics .PMBDLC designs, Wind Energy, Microprocessor based system design, Image and Video processing and controller area networks. 